\title{
Exploring Speech-Enabled Dialogue with the Galaxy Communicator Infrastructure
}

\author{
Samuel Bayer \\ The MITRE Corporation \\ 202 Burlington Rd. \\ Bedford, MA 01730 \\ sam@mitre.org
}

\author{
Christine Doran \\ The MITRE Corporation \\ 202 Burlington Rd. \\ Bedford, MA 01730 \\ cdoran@mitre.org
}

\author{
Bryan George \\ The MITRE Corporation \\ 11493 Sunset Hills Rd. \\ Reston, VA 20190 \\ bgeorge@mitre.org
}

\begin{abstract}
This demonstration will motivate some of the significant properties of the Galaxy Communicator Software Infrastructure and show how they support the goals of the DARPA Communicator program.
\end{abstract}

\section{Keywords}

Spoken dialogue, speech interfaces

\section{INTRODUCTION}

The DARPA Communicator program [1], now in its second fiscal year, is intended to push the boundaries of speechenabled dialogue systems by enabling a freer interchange between human and machine. A crucial enabling technology for the DARPA Communicator program is the Galaxy Communicator software infrastructure (GCSI), which provides a common software platform for dialogue system development. This infrastructure was initially designed and constructed by MIT [2], and is now maintained and enhanced by the MITRE Corporation. This demonstration will motivate some of the significant properties of this infrastructure and show how they support the goals of the DARPA Communicator program.

\section{HIGHLIGHTED PROPERTIES}

The GCSI is a distributed hub-and-spoke infrastructure which allows the programmer to develop Communicator-compliant servers in $\mathrm{C}, \mathrm{C}++$, Java, Python, or Allegro Common Lisp. This system is based on message passing rather than CORBA- or RPC-style APIs. The hub in this infrastructure supports routing of messages consisting of key-value pairs, but also supports logging and rule-based scripting. Such an infrastructure has the following desirable properties:

- The scripting capabilities of the hub allow the programmer to weave together servers which may not otherwise have been intended to work together, by rerouting messages and their responses and transforming their keys.

- The scripting capabilities of the hub allow the programmer to insert simple tools and filters to convert data among formats.

- The scripting capabilities of the hub make it easy to modify the message flow of control in real time.

- The scripting capabilities of the hub and the simplicity of message passing make it simple to build up systems bit by bit.

- The standard infrastructure allows the Communicator program to develop platform- and programminglanguage-independent service standards for recognition, synthesis, and other better-understood resources.

- The standard infrastructure allows members of the Communicator program to contribute generally useful tools to other program participants.

This demonstration will illustrate a number of these properties.

\section{DEMO CONFIGURATION AND CONTENT}

By way of illustration, this demo will simulate a process of assembling a Communicator-compliant system, while at the same time exemplifying some of the more powerful aspects of the infrastructure. The demonstration has three phases, representing three successively more complex configuration steps. We use a graphical display of the Communicator hub to make it easy to see the behavior of this system.

As you can see in Figure 1, the hub is connected to eight servers:

- $\quad$ MITRE's Java Desktop Audio Server (JDAS)

- $\quad$ MIT SUMMIT recognizer, using MIT's Mercury travel domain language model

- CMU Sphinx recognizer, with a Communicator-compliant wrapper written by the University of Colorado Center for Spoken Language Research (CSLR), using CSLR's travel domain language model

- A string conversion server, for managing incompatibilities between recognizer output and synthesizer input

- CSLR's concatenative Phrase TTS synthesizer, using their travel domain voice 
- $\mathrm{CMU} /$ Edinburgh Festival synthesizer, with a

Communicator-compliant wrapper written by CSLR, using CMU's travel domain language model for Festival's concatenative voice

- $\quad$ MIT TINA parser, using MIT's Mercury travel domain language model

- $\quad$ MIT Genesis paraphraser, using MIT's Mercury travel domain language model

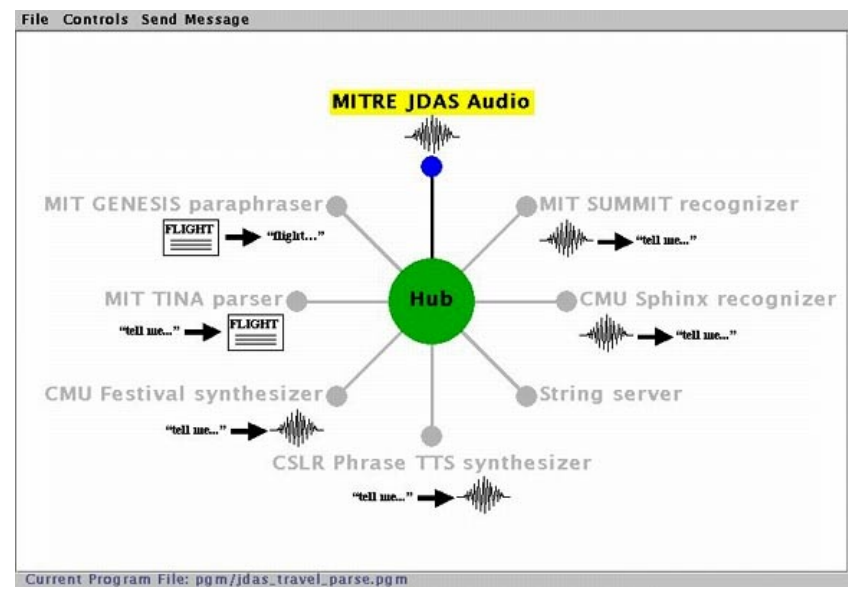

Figure 1: Initial demo configuration

We will use the flexibility of the GCSI, and the hub scripting language in particular, to change the path that messages follow among these servers.

\subsection{Phase 1}

In phase 1, we establish audio connectivity. JDAS is MITRE's contribution to the problem of reliable access to audio resources. It is based on JavaSound 1.0 (distributed with JDK 1.3), and supports barge-in. We show the capabilities of JDAS by having the system echo the speaker's input; we also demonstrate the barge-in capabilities of JDAS bye showing that the speaker can interrupt the playback with a new utterance/input. The goal in building JDAS is that anyone who has a desktop microphone and the Communicator infrastructure will be able to use this audio server to establish connectivity with any Communicator-compliant recognizer or synthesizer.

\subsection{Changing the message path}

The hub maintains a number of information states. The Communicator hub script which the developer writes can both access and update these information states, and we can invoke "programs" in the Communicator hub script by sending messages to the hub. This demonstration exploits this capability by using messages sent from the graphical display to change the path that messages follow, as illustrated in Figure 2. In phase 1, the hub script routed messages from JDAS back to JDAS (enabled by the message named "Echo"). In the next phase, we will change the path of messages from JDAS and send them to a speech recognizer.

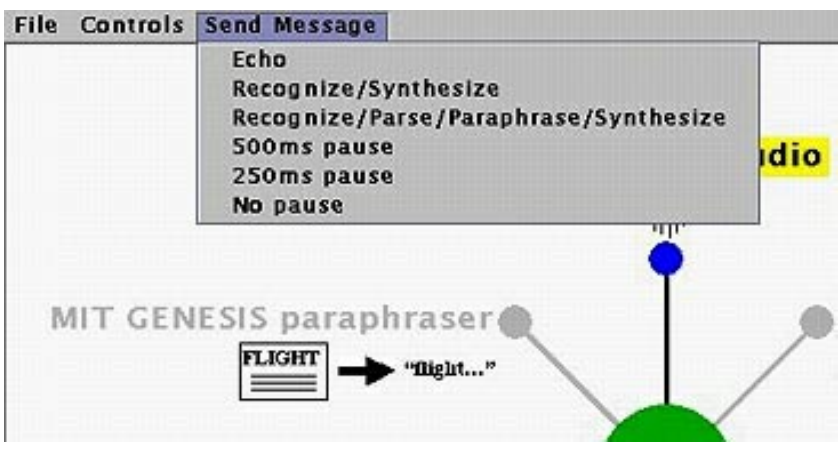

Figure 2: Modifying the hub information state

\subsection{Phase 2}

Now that we've established audio connectivity, we can add recognition and synthesis. In this configuration, we will route the output of the preferred recognizer to the preferred synthesizer. When we change the path through the hub script using the graphical display, the preferred servers are highlighted. Figure 3 shows that the initial configuration of phase 2 prefers SUMMIT and Festival.

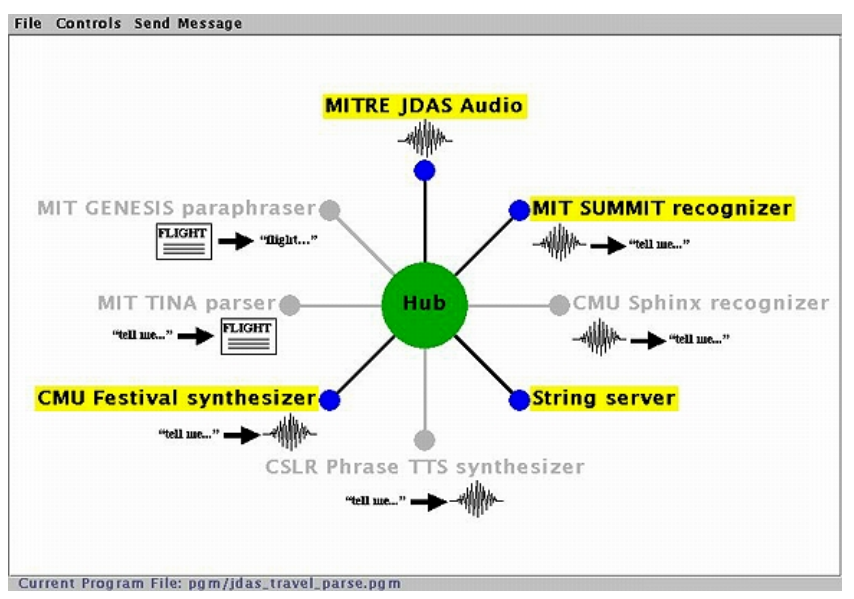

Figure 3: Initial recognition/synthesis configuration

The SUMMIT recognizer and the Festival synthesizer were not intended to work together; in fact, while there is a good deal of activity in the area of establishing data standards for various aspects of dialogue systems (cf. [3]), there are no programming-language-independent service definitions for speech. The hub scripting capability, however, allows these tools to be incorporated into the same configuration and to interact with each other. The remaining incompatibilities (for instance, the differences in markup between the recognizer output and the input the synthesizer expects) are addressed by the string server, which can intervene between the recognizer and synthesizer. So the GCSI makes it easy both to connect a variety of tools to the hub and make them interoperate, as well as to insert simple filters and processors to facilitate the interoperation.

In addition to being able to send general messages to the hub, the user can use the graphical display to send messages associated with particular servers. So we can change the preferred recognizer or synthesizer. (as shown in Figure 4), or change the Festival voice (as shown in Figure 5). All these messages are configurable from the hub script. 


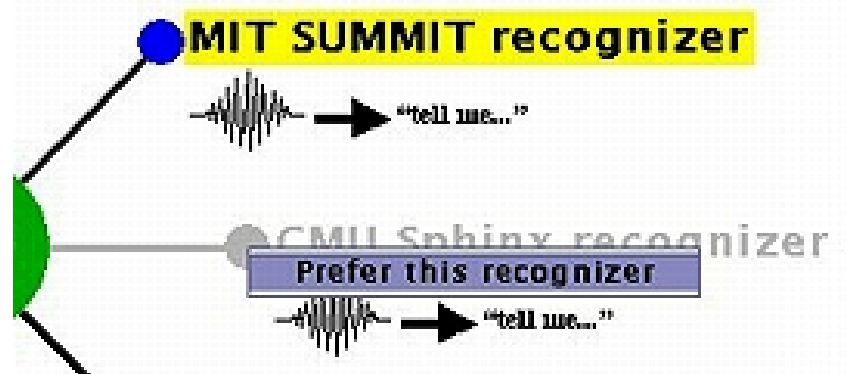

String server

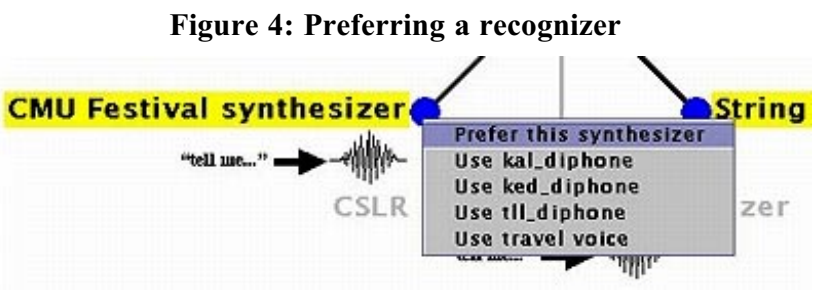

Figure 5: Changing the Festival voice

\subsection{Phase 3}

Now that we've established connectivity with recognition and synthesis, we can add parsing and generation (or, in this case, input paraphrase). Figure 6 illustrates the final configuration, after changing recognizer and synthesizer preferences. In this phase, the output of the recognizer is routed to the parser, which produces a structure which is then paraphrased and then sent to the synthesizer. So for instance, the user might say "I'd like to fly to Tacoma", and after parsing and paraphrase, the output from the synthesizer might be "A trip to Tacoma".

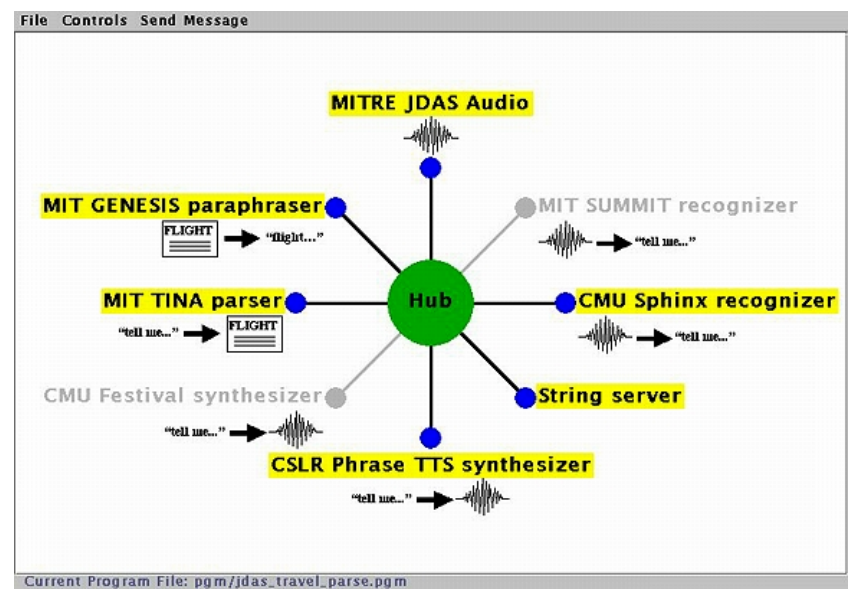

Figure 6: Adding parsing and paraphrase

\section{CONCLUSION}

The configuration at the end of phase 3 is obviously not a complete dialogue system; this configuration is missing context management and dialogue control, as well as an application backend, as illustrated by the remaining components in white in Figure 7. However, the purpose of the demonstration is to illustrate the ease of plug-and-play experiments within the GCSI, and the role of these capabilities to assemble and debug a complex Communicator interface. The GCSI is available under an open source license at http://fofoca.mitre.org/download.

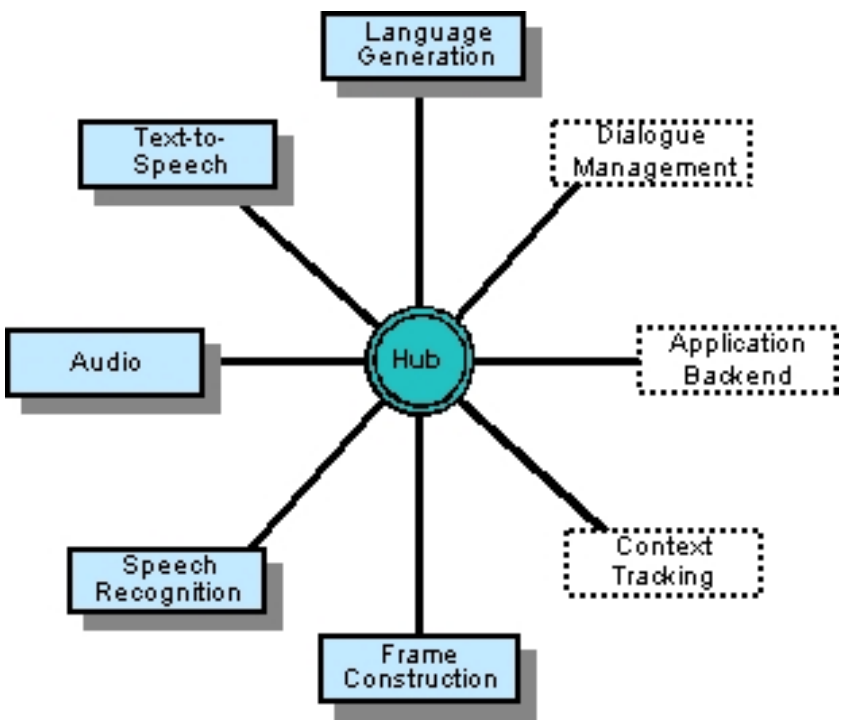

Figure 7: A sample full dialogue system configuration

\section{ACKNOWLEDGMENTS}

This work was funded by the DARPA Communicator program under contract number DAAB07-99-C201. (C) 2001 The MITRE Corporation. All rights reserved.

\section{REFERENCES}

[1] http://www.darpa.mil/ito/research/com/index.html.

[2] S. Seneff, E. Hurley, R. Lau, C. Pao, P. Schmid, and V. Zue. Galaxy-II: A Reference Architecture for Conversational System Development. Proc. ICSLP 98, Sydney, Australia, November 1998.

[3] "'Voice Browser' Activity." http://www.w3.org/Voice. 